\title{
Neurology postgraduate training: what is to be done?
}

\section{Adrian J Wills}

\section{The current system of teaching remains flawed}

See Editorial Commentary, p 1516

l: 1992, Kenneth Calman chaired a working group to reform British postgraduate specialist training. These reforms included a reduction in the duration of specialist training, the replacement of the old senior registrar and registrar grades by a combined specialist registrar grade (SpR), and the creation of a certificate of completion of specialist training (CCST), which would be recognised in all member states of the European Union. This latter recommendation implied the creation of objective setting, induction at the start of any training programme, training agreements, and rotational placements. The CCST would only be awarded once the trainee had "completed specialist training, based on assessment of competence, to a standard compatible with independent practice." How these parameters might be measured was not strictly defined, and differing systems were subsequently organised by the various colleges and specialist societies. No additional resources were made available to NHS Trusts for their implementation and no mandatory training programmes were introduced to ensure that the trainers were competent in the delivery of postgraduate medical education. ${ }^{1}$

It has been assumed that these reforms were designed to improve the quality of existing training programmes. However, it could be argued that the main aim of this restructuring was to develop a system of training more akin to existing models within the European Union. In 1992, before the publication of the Calman recommendations, there was great media interest in the "Goldstein" case, where a Harley Street rheumatologist had been denied inclusion on the specialist register in spite of holding a European community specialist certificate.

The Department of Health document "Hospital Doctors: Training for the Future" was published in April 1993. The working group responsible for producing this report was specifically requested by the Secretary of State for Health to advise her on "any action needed to bring the UK into line with
EC directives on medical training." Indeed, the European Commission had already decided to initiate infraction proceedings against the UK. This may account for why the reports' recommendations were quickly implemented and the necessary legislation enacted "without delay." It was also recognised that, in contrast to the old senior registrar grade, the new system would identify a clear end point to specialist training.

The publication of the Calman recommendations preceded high profile media and public criticism of the apparent failure of medical self regulation (Shipman, the Bristol Heart Inquiry). However, these reforms can be seen as part of a general trend to establish medical structures which were accountable and built on public trust. Indeed, the Calman report included specific statements about the need for NHS management to advise the Medical Royal Colleges "about the needs of the NHS and that training in the NHS continued to be delivered to the required standard." Although almost forgotten now, there had been a series of medical scandals in the 1980s, exemplified by the Wendy Savage case, which highlighted many issues surrounding the principles of competence and public trust. $^{2}$

These reforms can also be interpreted as part of a general response to the problem of the erosion of public trust in the medical profession and growing doubts about the appropriateness of self regulation. ${ }^{3}$ This was reflected by various trends including the rise of consumerism, a general decline in the cultural authority of the medical profession, and the massive increase in complaints or legal action about the standards of medical care. The NHS was founded on the basis of a socialist vision of society with paternalism (care from cradle to grave) at the core of its value system. Bevan's defining statement was that "No society can call itself civilised if a sick person is denied aid because of a lack of means." This model of health care is seen as increasingly anachronistic in a society structured on reverence for the free market. Thus far, public interest has concentrated on the regulation of performance rather than research or teaching, but it is highly likely that in future poorly trained or unsupervised junior doctors will reduce public trust and provoke a widening of the debate. The recent conviction of a middle grade junior doctor on a charge of manslaughter, associated with much lesser degrees of punishment inflicted on his seniors and hospital management, probably reflects this widening of scrutiny. ${ }^{4}$

\section{THE CALMAN REFORMS}

It was envisaged that the Calman reforms, which were introduced into all specialties by 1997, would lead to structured teaching and supervised learning with formal induction and regular appraisal. Each specialty would have a more clearly defined core curriculum, and competency would be assessed at least on an annual basis (by a record of in-training assessment (RITA)) before trainees could progress through the system. In addition trainees would receive regular advice and support from an educational supervisor (usually a consultant working in the same department) by a system of regular appraisal. Some specialties, particularly surgery and anaesthetics, modified pre-existing examination systems to allow the creation of an "exit exam" which had to be passed before a CCST in that particular specialty could be awarded. Most medical specialties have not thus far adopted this system.

In the penultimate year before accreditation a penultimate year assessment (PYA) takes place whereby each trainee is rigorously assessed, usually by an external assessor nominated by the Royal College of Physicians under the auspices of the Specialist Advisory Committee (SAC) of the medical Royal Colleges answerable to the Joint Committee on Higher Medical Training (JCHMT) (see later). The purpose of the PYA is to assure competency at that stage and ensure that the remaining training requirements can be achieved in the penultimate trainee year. Although in theory a panel consisting of a representative of the postgraduate dean, the regional specialty training committees, and the SAC should carry out all assessments, in practice this often only occurs on the occasion of the PYA. The final or exit RITA allows the award of CCST and inclusion on the specialist register.

The Calman reforms have also provided a template to assist trainees in difficulty. A RITA C is an annual record of satisfactory progress within the SpR grade. However, targeted training can also be recommended (RITA D) or even 
intensified supervision or repeat experience (RITA E). In addition, a formal appeals process exists to try and circumvent the theoretical risks of victimisation and bullying.

The implementation of these reforms has undoubtedly created an increased training workload for consultants. Each trainee has an educational supervisor who provides confidential advice and support during the training programme. In addition there is a nominated training programme director for each specialty, whose job is to ensure that curricular needs and JCHMT requirements are being met and that the annual assessments are well organised and fair. Interestingly, no safeguards have been introduced to assure the competence of either educational supervisors or training programme directors. The Standing Committee on Postgraduate Medical Education (SCOPME) has made recommendations to try and improve the teaching of postgraduate educational methods to hospital consultants, but this is still an area of concern. However, it was suggested in a recent publication that positive feedback, building trust, and assessing the trainees' learning requirements are all areas that could be markedly improved within the postgraduate medical education system. ${ }^{5}$

\section{NEUROLOGY POSTGRADUATE TRAINING (HMT) AND THE CALMAN REFORMS}

Before entry to the neurology SpR grade all trainees must spend at least two years in general professional training, which should provide a broad experience of medicine at senior house officer (SHO) grade. During this period trainees must also acquire membership of the Royal College of Physicians (MRCP) or equivalent.

The duration of higher medical training (HMT) in neurology is five years. Most rotations are based in a large neuroscience centre but trainees usually spend at least one year either at a district general hospital (DGH) or at another teaching hospital in the same Region. This allows exposure to a larger number of consultants and thus a variety of teaching styles. Currently, entry to the SpR grade is extremely competitive and most applicants will have completed a period of research and registered for an $\mathrm{MD}$ or $\mathrm{PhD}$ before taking up an SpR post.

The neurology curriculum is defined in a document (Curriculum for Higher Specialist Training in Neurology) which is regularly updated by the JCHMT. Earlier versions of this document (1996 onwards) merely listed various areas in which the trainee was expected to have "obtained experience." How this was to be done and the expected frequency or intensity of exposure to any particular subsection of the curriculum was not defined. Several "core" subjects were included within the curriculum, encompassing clinical neurophysiology, rehabilitation medicine, neurosurgery, intensive care, neuroradiology, head injury, clinical audit, and research. In addition the trainee was expected to obtain some experience in, among others, neuro-ophthalmology, neurootology, neuropaediatrics, and pain management. Various "domains" within the field (such as neuropsychiatry, disorders of the cranial nerves, and neurotoxicology) were also listed, and trainees were advised to have "attended teaching sessions and read widely" on these subjects.

The 1996 version of the curriculum for higher specialist training in neurology ran to only 12 pages and provided few specific recommendations as to how training requirements were to be met. Later versions of this document have been amended to provide more specific instructions. Thus, for example, a minimum number of EEGs which the trainee is expected to have reported has been recommended to assure standards in that particular area of the curriculum. In addition, each part of the curriculum has been listed, together with a set of learning objectives and methods. Specific details are also given which explain how assessment is undertaken and the form whereby competence in the particular subject should be recorded. The 2003 version of the curriculum runs to 53 pages and, in contrast with earlier versions, does seem to have been influenced by modern teaching theory. Various assessment methods are being piloted by the JCHMT in collaboration with the education department of the Royal College of Physicians of London. These include MiniCEX (mini clinical evaluation exercises), DOPS (directly observed procedural skills), and 360 degree assessment. One or all of these methods will presumably be introduced as an assessment tool in the near future.

The current delivery of neurology postgraduate education takes several forms. There is still heavy reliance on bedside teaching through the mechanism of the consultant ward round and learning by an "apprenticeship" system. Trainees are expected to undertake private study and protected time is allocated for this purpose. In the past the attainment of neurological consultation skills has always been assumed en passant, but now a variety of assessment tools has been introduced. These include videotaping of SpR interviews with real patients and subsequent analysis and feedback from an educational supervisor. Simulated consultations have also been piloted to develop communication skills in neurological trainees. ${ }^{6}$

Regular (once monthly) training days have also been introduced, orientated around a particular topic. The main teaching methods used include lecturing and small group teaching. However, aims and objectives are rarely defined and learning outcomes rarely measured, bar recording of attendance in the trainees' logbook. Problem based learning is very rarely used, perhaps because it is difficult within the constraints of trainees' and consultants' service commitments to ensure that participants can regroup on a number of separate occasions. This is in contrast to some European training programmes, where problem based learning approaches have been validated and seem to be highly effective..$^{78}$ It is most unusual for ground rules to be established, and concepts such as buzz groups, pyramids, and role-play are rarely developed.

\section{NEUROLOGICAL POSTGRADUATE TRAINING IN THE PRE-CALMAN ERA}

It is sobering to realise that arrangements for specialist medical training were not even discussed until 1969. The first meeting of the JCHMT took place in 1970. The responsibilities of this committee were to formulate guidelines for training in medical specialties and to approve posts and training programmes as well as grant certificates of accreditation (from 1977 onwards). Intriguingly, as recently as 1992 accreditation was not considered mandatory for appointment to a consultant post. SACs were also created, whose role was to advise the JCHMT on the nature of training requirements in individual specialties.

Postgraduate training was envisaged as containing the following elements: a preregistration year, three years' general professional training, higher specialist training, and "continuing education as a normal part of professional life." How this latter proposal was to be policed was never defined.

It was envisaged that general professional training could include some time at registrar level (usually in a general medical role). Trainees were expected to have obtained MRCP before entry to specialist training. The JCHMT maintained a list of approved training posts and carried out infrequent inspections to ensure that standards were maintained. Approved posts were expected to provide increasing clinical autonomy with increasing seniority. Trainees were supposed to devote half their time to 
clinical work, with the remaining 50\% split between teaching and research. Senior registrar posts could be approved to a maximum of five years, although this period was often extended routinely when consultant jobs were scarce. Although, theoretically, accreditation was not automatically consequent upon enrolment, in practice it was. Trainees were supposed to spend a minimum of two years at senior registrar level.

In contrast with the modern era, the neurology curriculum for higher specialist training circa 1992 occupies half a side of A4 paper. A period of four years was to be spent at senior registrar level, but trainees could gain retrospective recognition for a maximum period of two years spent at registrar level or in research. However, in practice, owing to the small numbers of consultant neurology posts in the UK at that time, it was not uncommon for the training period to be much more prolonged. Curricular requirements were succinctly summarised in the following statement: "It is desirable that trainees should have obtained experience in neurophysiology, rehabilitation, neurosurgery, intensive care, and in some of the following: neuropaediatrics, neuro-ophthalmology, neuroradiology, head injury, pain management, spinal injury, neuropathology, genetics and clinical audit"!

\section{HAVE THE CALMAN REFORMS IMPROVED NEUROLOGY POSTGRADUATE EDUCATION?}

The current system of training undoubtedly places increased emphasis on structured teaching and supervised learning and less on experiential apprenticeship. However, the length of the training programme has been reduced (by two years on average) and the greater degree of autonomy encouraged by working arrangements in the old senior registrar grade may have had demotivating effects, particularly with regard to self directed learning. The whole issue of whether the current system creates better educated doctors is fraught with difficulties. There are probably too many extraneous factors in operation, such as the reduction in junior doctors' hours and the increasing demand for a consultant led service, to allow this problem to be answered with any confidence. In continental Europe, masters degree courses have been developed based on an integrated network of training programmes. ${ }^{9}$ This approach might offer a more effective means of coordinating neurology training between participating nations.

Paice and coworkers did evaluate the impact of the Calman reforms on specialist training using a questionnaire administered to over 3000 trainees in several specialties. They concluded that trainees in all grades reported greater satisfaction with the new system. ${ }^{10}$ It could also be argued that the creation of a CCSTimplying structured training and assessment-provides greater public reassurance in contrast to the previous system of training. Further research is urgently required to determine whether calmanisation has created higher standards, which might be reflected by a decrease in complaints and legal claims.

\section{CONCLUSIONS}

Neurological conditions account for $28 \%$ of all years of life lived with disability. ${ }^{11}$ Neurological education is therefore an extremely important part of the medical curriculum. However, the current system of teaching is still flawed and it is debatable whether the Calman reforms have improved matters to any great extent. It is tempting to analyse the reasons for these failures and to speculate on how matters could be improved.

Most medical students feel that the theoretical underpinning of neurology is more difficult to conceptualise than that of other specialties. One of the major reasons identified in a large survey of medical students and junior doctors was poor quality and inadequate teaching. ${ }^{12}$ A significant proportion of teaching at undergraduate level is delivered by consultant neurologists who are also responsible for delivering the postgraduate course and ensuring that postgraduate training contains the necessary elements specified in the JCHMT curriculum. There is a strong argument that amateur or part time teachers are inadequately equipped for this task. If it is true that there are "fewer born teachers than born poets", this suggests that a radical overhaul of the current system is in order. It could be argued that medical teachers should be salaried and subject to regular appraisal or performance rating. The qualifications for membership of curricular committees (such as the Training and Education Sub-Committee (TESC) of the Association of British Neurologists) seem to be rather haphazard and it could be mandatory for applicants to have a formal teaching qualification. These changes would have important resource implications, increasing the already substantial cost of postgraduate training. ${ }^{13}$

The loss of the old senior registrar grade, which undoubtedly did encourage self directed learning and promoted autonomy, is regrettable. It is evident that in spite of JCHMT recommendations, feedback and induction mechanisms for neurology specialist registrars could be improved. Objective setting on induction needs to be more clearly defined and assessment processes more rigorously enforced. The introduction of the "miniCEX" and "DOPS" should encourage competency based assessment of clinical skills and practical procedures, respectively. It is also self evident that unless there is a major expansion of consultant numbers in the UK none of these measures will be achieved and training will continue to be haphazard while service commitments are so onerous for the majority of consultants. The true costs of medical education are unlikely to remain covert under the terms of the new consultant contract. It is sobering to compare the recommended active involvement in patient care for Dutch neurologists with that of their UK counterparts (16 versus 30 hours per week). ${ }^{14}$ Unless our political masters address these resource shortfalls it is unlikely that neurological postgraduate education in the UK will improve to any great extent in the foreseeable future.

J Neurol Neurosurg Psychiatry 2004;75:1513-1515.

doi: 10.1136/jnnp.2004.040725

\section{Authors' affiliations}

A J Wills, Department of Neurology, Queen's Medical Centre, Nottingham, UK

Correspondence to: Dr A J Wills; adewills61@ hotmail.com

\section{REFERENCES}

1 Catto G. Specialist registrar training. Some good news at last. BMJ 2000:320:817-18.

2 Inch S. Professional incompetence. Lancet 1986; ;:864-5

3 Salter B. Who rules? The new politics of medical regulation. Soc Sci Med 2001;52:871-83.

4 Dyer C. Doctors face trial for manslaughter as criminal charges against doctors continue to rise. BMJ 2002;325:63.

5 Wall D, McAleer S. Teaching the consultant teachers: identifying the core content. Med Educ 2000:34:131-8.

6 Smith PE, Fuller GN, Kinnersley P, et al. Using simulated consultations to develop communications skills for neurology trainees. Eur J Neurol 2002:9:83-7.

7 Smits PB, de Buisonje CD, Verbeek JH, et al. Problem-based learning versus lecture-based learning in postgraduate medical education. Scand J Work Environ Health 2003;29:280-7.

8 Stromso HI, Grottum P, Hofgaard Lycke K. Changes in student approaches to learning with the introduction of computer-supported problem based learning. Med Educ 2004;38:390-8.

9 Cavallo F, Rimpela A, Normand C, et al. Public health training in Europe. Development of European masters degrees in public health. Eur J Public Health 2001;11:171-3.

10 Paice E, Aitken M, Cowan G, et al. Trainee satisfaction before and after the Calman reforms of specialist training: questionnaire survey. $B M J$ 2000;320:832-6.

11 Menken M. Demystifying neurology. BM 2002;324:1469-70.

12 Schon F, Hart P, Fernandez C. Is clinical neurology really so difficult? I Neurol Neurosurg Psychiatry 2002;72:557-9

13 Babineau TJ, Becker J, Gibbons G, et al. The "cost" of operative training for surgical residents. Arch Surg 2004; 139:366-9.

14 Keyser A. Development of teaching and tuition in the specialty of neurology in the Netherlands. Eur J Neurol 2003;10:343-51. 
The main point would be the need to Neurology postgraduate training: what
is to be done?

\section{Giroud}

\section{Improving neurology postgraduate training}

n the paper by A J Wills (page 1513, this issue), an evaluation of the results of the Calman reforms on medical postgraduate training in the UK is reported. The Calman reforms were introduced in the UK in 1997 and the current evaluation is useful from both a UK and a European perspective.

The objectives of the reforms were to improve the structure, and to supervise the quality of medical learning, competency, and training programmes. The reforms were developed in response to changes in Public Health policy, to public criticism, and to a perceived erosion of trust in the medical profession. The first consequence of this project was to improve the training of junior doctors, which led to structured teaching and supervised learning. Each specialty now has a more clearly defined core curriculum, trainees can receive regular advice and support from an educational supervisor, and accreditation is given after evaluation of competency. The reforms improved, standardised, and coordinated teaching, and supervised learning to encourage autonomy in self learningleading to better theoretical learning and practice. Neurology training was structured according to these reforms with a 5 year higher medical education. A curriculum is defined in a document that is regularly updated, includes the subspecialties of the neurological field, and relies on rotations between several teaching hospitals.

These reforms could be generalised within the European Union leading to several recommendations. The first advantage of these reforms would be to standardise European medical training, allowing mobility of medical trainees and doctors within Europe. The medical training could be adapted to the new European Master Programme. New learning methods ${ }^{2-4}$ would be developed as well as problem based learning, e-learning, video taping, and simulated consultations. full time qualified medical professionals and medical teachers. The formal qualification of medical teachers-with an evaluation-would be generalised and standardised within European Union.

In addition, two other criteria are very important: (i) the development of self training could be an excellent way to improve autonomy and to continue medical training; (ii) research programmes would be developed during medical training because research is critical for medical progress and quality of care. Such reforms would carry a financial cost but would and also require a cultural change.

J Neurol Neurosurg Psychiatry 2004;75:1516. doi: 10.1136/jnnp.2004.048462

Correspondence to: $M$ Giroud, Dean of the Faculty of Medicine, Boulevard Jeanne D'Arc, 21000 Dijon, France; maurice.giroud@ chu-dijon.fr

\section{REFERENCES}

1 Wills AJ. Neurology postgraduate training: what is to be done? J Neurol Neurosurg Psychiatry 2004;75:1513-15.

2 Cruess SR, Johnston S, Cruess RL. Professionalism: a working definition for medical educators. Teach Learn Med 2004;16:90-2.

3 Miflin BM, Campbell CG, Price DA. A conceptual framework to guide the development of self-directed, lifelong learning in problembased medical curricula. Med Educ 2000;34:299-306.

4 Stromso, et al. Changes in Student approaches to learning with the introduction of computersupported problem-based learning. Med Educ 2004;38(4):340-8.

\section{Enlarged Virchow-Robin spaces: do they matter?}

\section{F Barkhof}

\section{The clinical significance of widened Virchow-Robin spaces}

$\mathrm{W}$ ith the introduction of magnetic resonance imaging (MRI), we have become increasingly aware of the process of brain maturation and ageing. While there is MRI evidence that the former may continue into the third decade, the latter starts as early as the fourth decade. Early ageing phenomena include the development of subtle loss of brain tissue with widening of the sulci and ventricular system, development of periventricular caps and bands, and dilatation of the perivascular VirchowRobin spaces (VRS).

The VRS are extensions of the subarachnoid space that accompany vessels entering the brain parenchyma. Widening of VRS often first occurs around penetrating arteries in the substantia perforata and can be seen on transverse MRI slices around the anterior commisure, even in young subjects. Another typical location of widened VRS is near the vertex of the brain, but with advancing age they can be seen anywhere in the white matter, basal ganglia, and hippocampus, especially when high resolution (using a 512 matrix) images are obtained. Their typical MRI appearance includes sharp demarcation and cerebral spinal fluid (CSF)-like signal on all sequences-depending on location and slice orientation, they can appear as dots or linear structures (fig 1).

In this issue, MacLullich et al ${ }^{1}$ (see page 1519) examine the clinical significance of widened VRS in a community-based sample of 79 healthy men. A standard MRI protocol was used with a $1 \mathrm{~mm}$ inplane resolution and a categorical scoring system was devised to try to account for the multiplicity of widened VRS. The lowest score (less than 10 widened VRS) was found in the majority of subjects. Higher VRS scores were associated with decreased performance in terms of nonverbal reasoning and general visuospatial ability. However, when controlling for the coexistence of T2-hyperintense 

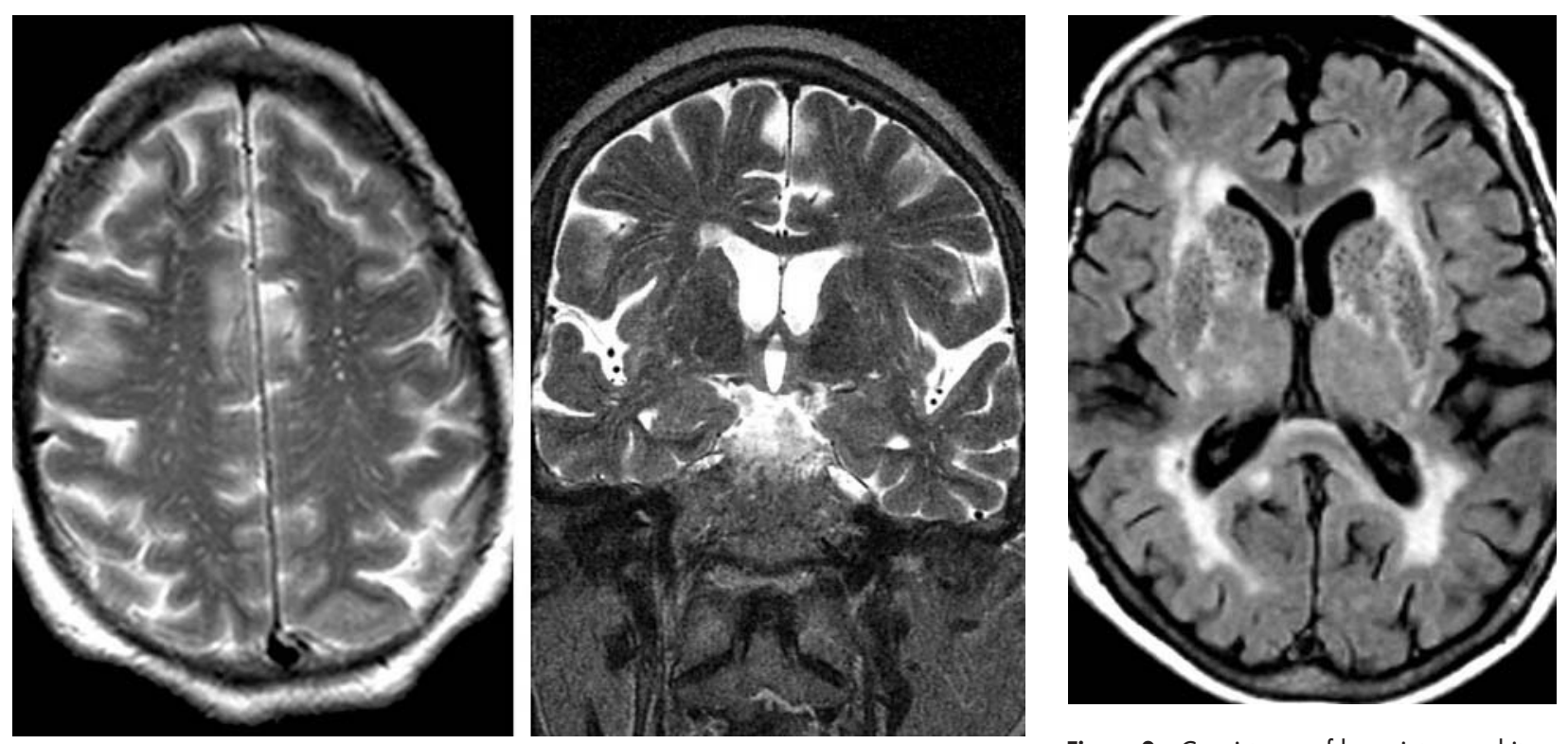

Figure 1 Heavily T2-weighted MRI obtained with high resolution, showing multiple enlarged VRS, visible as well-demarcated CSF like structures, which, dependent on their orientation and plane of imaging, appear as dots (transverse image on the left) or stripes (coronal image on the right).

white matter lesions (WML), VRS did not seem to have an independent contribution to (decreased) cognitive performance. VRS were also rated separately in the hippocampal region, and although these were significantly correlated with WML, they were not associated with poorer cognitive performance (including episodic memory).

It is tempting to speculate on the pathogenesis of widened VRS and their significance in determining white matter integrity. Clearly, these CSF spaces do not represent viable tissue, and thus present (a mild form of) local atrophy, which may occur independent of cortical atrophy and ventricular widening. In my clinical experience, widened VRS in elderly subjects often coincide with WML on MRI, and this corroborates with the current findings of MacLullich et al. Apparently, white matter damage may manifest itself as general atrophy (with ventricular widening), incomplete white matter infarction (with WML on MRI), and by virtue of widened VRS. What is particularly interesting is why some patients may develop extensive WML in the basal ganglia and diffusely widened VRS (so-called état criblé) without significant atrophy (fig 2), while others develop only volume reduction (global atrophy). Perhaps this reflects a different mode of communication of the VRS with the subarachnoid space. This remains a subject of debate.

In summary, the findings by MacLullich et al indicate that widened VRS are a common ageing phenomenon that is associated with WML and cognitive function. More work is needed to develop an integrated methods to probe
Figure 2 Coexistence of hyperintense white matter lesions (WML), and diffusely widened enlarged hypointense VRS (so-called état criblé) on a CSF-supressed transverse MR image.

white matter integrity, which should not only address WML, but also the quality of the remaining tissue (e.g. using diffusion tensor MRI), and residual white matter volume by accounting for the degree of widening of the VRSa measure of focal atrophy.

J Neurol Neurosurg Psychiatry 2004; 75:1516-1517.

doi: 10.1136/jnnp.2004.044578

Correspondence to: F Barkhof, Department of Radiology, Image Analysis Centre, and Alzheimer Centre, VU Medical Centre, PO Box 7057, 1007 MB Amsterdam, The Netherlands; f.barkhof@vumc.nl

\section{REFERENCE}

1 MacLullich AMJ, Wardlaw JM, Ferguson KJ, et al. Enlarged perivascular spaces are associated with cognitive function in healthy elderly men. J Neurol Neurosurg Psychiatry 2004;75:1519-23.

\section{Longer term outcome of children born to mothers with epilepsy}

\section{S D Shorvon}

\section{New issues concerning valproate treatment during pregnancy}

$\mathrm{T}$ he teratogenic risk of antiepileptic drugs has been a clinical concern for at least three decades. In an ideal world this risk would be balanced against the benefits of reduced foetal exposure to seizures due to the drug. In the real world, however, statistical data on both sides of this equation are limited. Although it has long been held that maternal seizures during pregnancy can damage the developing foetus, there is actually little hard data to support this view. Convulsive attacks certainly carry some risk-especially in later pregnancy-not least because of the mechanical dangers of convulsions, but the extent of the overall risk is unclear. It also seems inherently unlikely that the short lived anoxia occurring in seizures will have a profound effect on the foetus, although this has often been postulated. The risks of non-convulsive seizures or myoclonus are totally unknown but intuitively are likely to be slight. There is also considerable uncertainty about the extent of the 
teratogenic risk of antiepileptic drugs, and the best data concern the rates of major malformations. In recent years, a great deal of attention has focused on the use of the anticonvulsant sodium valproate (and its derivatives such as valproic acid). Valproate was first introduced into practice in 1961 but it was not until the early 1980s that the first reports of teratogenicity were made. It is now recognised that the drug confers an increased risk of neural tube defects (about $1-2 \%$ of pregnancies) and also of more minor dysmorphic features, the true incidence of which remains controversial. In an attempt to define teratogenicity rates more accurately, pregnancy registers have been set up in many countries in recent year, and overall malformation rates on valproate monotherapy and polytherapy of between about 3-15\% have been reported, with higher rates in polytherapy and at higher doses.

In this issue (pp 1575-83), an important and carefully argued paper ${ }^{1}$ is published that adds a new dimension to the problem. The study shows that 41 school aged children exposed to valproate monotherapy in utero have significantly lower verbal IQ (VIQ) scores when compared with 52 children exposed to carbamazepine and 21 to phenytoin monotherapy. In a multivariant analysis, low VIQ was also associated with the occurrence of five or more tonic-clonic seizures during pregnancy and a low maternal IQ. There were higher rates of dysmorphic features-defined as cosmetic variants without disability-in the valproate exposed children, and these were most common in those with low VIQ scores. These longer term developmental effects would not have been identified at birth and thus not in the pregnancy registers. This is a deeply worrying finding and come on the heels of parallel findings from other studies by the same group as well as others. ${ }^{2-4}$

Some caution needs to be exercised in interpreting these results. The study was a retrospective survey, the mothers were not randomised to different monotherapies, there was only a $40 \%$ response rate, and there are some inconsistencies (for instance, significant differences in VIQ rates were not found in foetuses exposed to valproate polytherapy and there was only a soft relationship with dose). A biological explanation of the findings would add plausibility, but is outwith the scope of the paper. The authors point out that for methodological reasons the risk is probably overestimated and that the absolute risk will be satisfactorily ascertained only by a prospective community-based study. However, with the publication of these findings such a study may prove difficult to justify and it is now quite possible that exact estimates of the risks of valproate will never be possible to ascertain.

What advice can we therefore give to patients? The authors conclude with a demand that epilepsy services to deliver adequate information and counselling, and who could argue with this? The problem is that available information is not adequate, particularly in patients with idiopathic generalised epilepsy where valproate is the drug of choice for seizure control. We should be grateful for Chadwick and colleagues for identifying what is potentially a major clinical concern and introducing the concept that antiepileptic drug teratogenicity may include long term effects on cognitive development. It is also notable that this effect has been only recently postulated in spite of the fact that valproate has been in use for over 40 years. Further exploration of this vital area is now urgently needed.

J Neurol Neurosurg Psychiatry 2004; 75:1517-1518.

doi: 10.1136/jnnp.2004.050641

Correspondence to: Professor Simon D Shorvon, Institute of Neruology, Queen Square, London WCIN 3BG, UK; s.shorvon@ion.ucl.ac.uk

\section{REFERENCES}

1 Adab Nm Kini U, Vinten J, et al. The longer term outcome of children born to mothers with epilepsy. J Neurol Neurosurg Psychiatry 2004;75:1575-83.

2 Gaily E, Kantola-Sorsa E, Hilesmaa V, et al. Normal intelligence in children with prenatal exposure to carbamazepine. Neurology 2004;62:28-32.

3 Eriksonn K, Viinikainen K, Monkkonen A, et al. The effects of Antiepileptic drug exposure in utero to neurological and cognitive functioning of children of school age. Epilepsia 2004;45(Suppl 3):57

4 Adab N, Jacoby A, Smith D, et al. Additional educational needs in children born to mothers with epilepsy. J Neurol Neurosurg Psychiatry 2001;70:15-21. 